\title{
Phillips-Perron-type unit root tests in the nonlinear ESTAR framework
}

\author{
Christoph Rothe ${ }^{1}$ \\ Philipp Sibbertsen ${ }^{2}$ \\ Diskussionspapier 315 \\ ISNN 0949-9962
}

Zusammenfassung: In dieser Arbeit schlagen wir semiparametrische Tests vor, um Prozesse mit Einheitswurzeln von mean-reverting ESTARModellen zu unterscheiden. Die Tests sind vom Typ des PhillipsPerron Tests. Die Grenzverteilung der Teststatistiken wird unter sehr allgemeinen Annahmen hergeleitet. Eine Simulationsstudie zeigt, dass die Tests im Bereich der Nullhypothese bessere Eigenschaften haben als der Standard Phillips-Perron Test oder der Dickey-Fuller Test.

\begin{abstract}
In this paper, we propose Phillips-Perron type, semiparametric testing procedures to distinguish a unit root process from a mean-reverting exponential smooth transition autoregressive one. The limiting nonstandard distributions are derived under very general conditions and simulation evidence shows that the tests perform better than the standard Phillips-Perron or Dickey-Fuller tests in the region of the null.
\end{abstract}

KEYWORDS: Exponential smooth transition autoregressive model; Unit roots; Monte Carlo simulations; Purchasing Power Parity

JEL-Classification: C 12; C 32

\footnotetext{
${ }^{1}$ Universität Dortmund - Fachbereich Statistik

${ }^{2}$ Corresponding Author:

Fakultät Wirtschaftswissenschaften, Abteilung Statistik

Königsworther Platz 1, 30167 Hannover

Fax-Nr. +49- 511- 7623923

E-Mail: sibbertsen@mbox.iqw.uni-hannover.de
} 


\section{Introduction}

The application of unit root tests, such as the Augmented Dickey-Fuller (ADF) and the Phillips-Perron (PP), has become standard in applied time series econometrics. In some areas of economics, however, their frequent inability to reject the null of nonstationarity contradicts well-established theoretical expectations.

One example is the purchasing power parity (PPP) hypothesis. In its absolute form, it states that a common basket of goods, when quoted in the same currency, costs the same in all countries. Due to several factors like taxes, tariffs, trade restrictions and other market imperfections, the relationship may only be expected to hold in the long run. In empirical studies, however, the null hypothesis of a unit root in the deviations from parity can often not be ruled out using ordinary testing procedures.

As a possible explanation, Dumas (1992), Sercu, Uppal and Van Hulle (1995) and Michael, Nobay and Peel (1997) consider models of real exchange rate determination which take transactions costs into account. Their theory suggests that the larger the deviation from PPP, the stronger the tendency to move back to equilibrium. The result is a nonlinear, mean-reverting stochastic process. They claim that standard unit root tests might lack power in such cases, and thus the evidence against PPP in the literature might be the result of a type II error.

It has been demonstrated by Michael et al. (1997), Sarno (2000), Taylor, Peel and Sarno (2001) and Baum, Barkoulas and Caglayan (2001), amongst others, that the exponential smooth transition autoregressive (ESTAR) model is an effective way to describe such processes. This is part of the general class of STAR models, which constitute a broad and flexible family of nonlinear time series models, that has recently been applied in various fields of economics. For an excellent survey, see van Dijk, Teräsvirta and Franses (2002).

The problem is that these authors assume stationarity a priori, without using formal inference. Therefore Kapetanios, Shin and Snell (2003) develop an ADF type test called $t_{N L}$ in that framework, and confirm its superiority over its linear counterpart in some situations. Our paper extends their work by proposing two new test statistics using a semi-parametric approach along the lines of Phillips (1987) and Phillips and Perron (1988). We give analytical expressions for the asymptotic distributions, which are free of nuisance parameters. By simulation, we also study the performance in small samples and compare it to both their linear counterparts and the $t_{N L}$.

The plan of the paper is as follows. Section 2 describes the theoretical framework and derives some important asymptotic results. In Section 3, the new tests are developed along with their limit distributions. Finite sample properties are assessed in Section 4, while an emprirical application is presented in Section 5. Finally, Section 6 concludes. A more technical theorem is given in the Appendix.

Some words on notation. Throughout the paper, $\|X\|_{r}=\left(E\left(|X|^{r}\right)\right)^{1 / r}$ de-

notes the $L_{r}$ norm, $W$ denotes a standard Wiener process on $[0,1]$, and " $\Rightarrow$ " denotes weak convergence in distribution. 


\section{The nonlinear ESTAR framework}

Consider the case of a simple STAR(1) model given by

$$
y_{t}=\phi_{1} y_{t-1}\left(1-G\left(y_{t-1}, \theta\right)\right)+\phi_{2} y_{t-1} G\left(y_{t-1}, \theta\right)+v_{t},
$$

where $G\left(y_{t-1}, \theta\right)$ is called the transition function, is continuous and bounded between 0 and 1 , and $\left\{v_{t}\right\}$ is a mean zero innovation sequence whose exact properties will be discusses in more detail below. The easiest way to think of such a nonlinear model is as a weighted average of two regimes corresponding to $\mathrm{AR}(1)$ processes, with dynamically changing weights determined by the level of the process in the previous period. To begin with, we will assume that $\left\{y_{t}\right\}$ is a mean zero stochastic process. Other cases will be discussed in Section 3 .

While there are various transition functions being considered in the literature, our focus will be on the exponential one, which is of the form

$$
G\left(y_{t-1}, \theta\right)=1-\exp \left(-\theta y_{t-1}^{2}\right),
$$

with $\theta \geq 0$. This function is symmetrically $\mathrm{U}$-shaped around zero and tends to 1 as $y_{t-1}$ gets large in absolute value, provided that $\theta$ is positive. In this case, the value of the $\theta$ determines the curvature of the function and thus effectively the speed of the transition between the two regimes. When $\theta=0$, however, the function is zero everywhere and no transition takes place.

For our particular application, we follow Kapetanios et al. (2003) and impose $\phi_{1}=1$, and $-1<\phi_{2}<1$. In other words, the two regimes of our STAR model are assumed to correspond to a unit root process and a stable $\operatorname{AR}(1)$, respectively.

Combining these restrictions with (1) and (2), we can write our model in the following, reparameterized form:

$$
\Delta y_{t}=\gamma y_{t-1}\left(1-\exp \left(-\theta y_{t-1}^{2}\right)\right)+v_{t},
$$

where $\gamma=\phi_{2}-1$. When $-2<\gamma<0$, which we assume holds, and $\theta>0$, the process is globally mean-reverting towards the long-run level zero, while it locally acts like a unit root process when being close to that same level. We will say that the time series has a partial unit root in this case. For $\theta=0$, there is no mean-reverting behaviour and the process has a full unit root.

It seems likely that standard linear unit root tests such as the ADF or PP have difficulties to distinguish between these two cases, i.e. they lack power against the nonlinear alternative described above. For the former test, this was shown by Kapetanios et al. (2003), while simulation evidence on the latter one is presented in Section 4 of this paper. We will therefore describe their approach to test for a unit root that takes the specific alternative into account.

The hypothesis is

$$
H_{0}: \theta=0 \quad \text { vs. } \quad H_{1}: \theta>0 .
$$

Note that the parameter $\gamma$ is not identified under the null. Loosely speaking, this means that nothing can be learned about $\gamma$ from the data if $H_{0}$ is actually true. This highly affects standard inferential procedures (see Davies (1987)), and thus a direct testing procedure is not feasible. However, following Luukkonen, Saikkonen and Teräsvirta (1988), the problem can be overcome by using a 


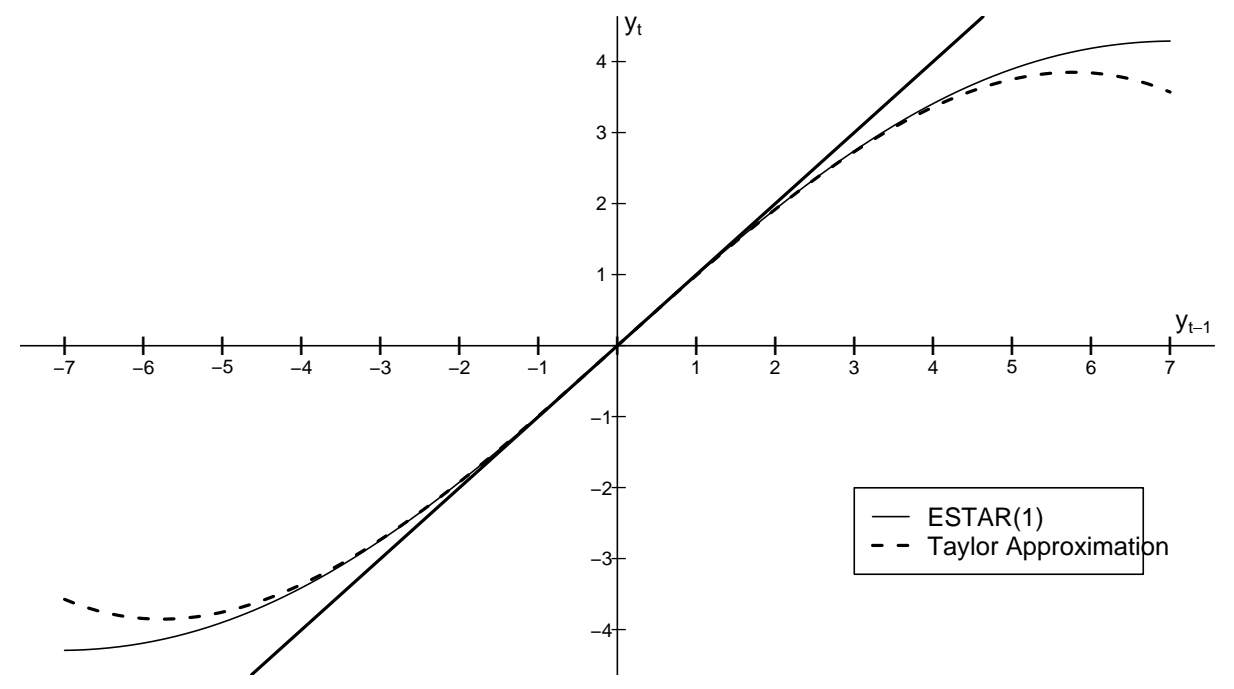

Figure 2.1: Undisturbed relationship between $y_{t}$ and $y_{t-1}$ for the ESTAR(1) model (3) and corresponding Taylor approximation (5), using $\gamma=-1, \theta=0.01$. The bisecting line represents a unit root process

first order Taylor series approximation of the exponential function around zero, leading to the auxiliary regression

$$
\Delta y_{t}=\delta y_{t-1}^{3}+u_{t}
$$

where $\delta=\theta \gamma, u_{t}=v_{t}+\gamma y_{t-1} R\left(y_{t-1}, \theta\right)$ and $R\left(y_{t-1}, \theta\right)$ is the remainder of the Taylor approximation. Here the identification problem is no longer present.

To get an idea of the goodness of the approximation, Figure 2.1 depicts the undisturbed relationship (i.e. without the error term) between consecutive observations implied by the respective regressions. As one can see, differences are only getting obvious when $y_{t-1}$ becomes large in absolute value. Auxiliary regression (5) therefore captures the dynamics of the true process quite well.

In the regression (5) our hypotheses stated above are equivalent to

$$
H_{0}: \delta=0 \quad \text { vs. } \quad H_{1}: \delta<0 .
$$

Now, the idea is to base inference on estimates of $\delta$ and the respective $t$ ratios. Note that although our regression is only an approximation, under the null hypothesis this does not influence the properties of the error term, since $R\left(y_{t-1}, \theta\right) \equiv 0$ and thus $u_{t}=v_{t}$ when $H_{0}$ is true. Consequently, it does not affect the asymptotic distribution theory under $H_{0}$.

Denote the ordinary least squares (OLS) estimate of $\delta$ by

$$
\hat{\delta}=\frac{\sum_{t=1}^{T} y_{t-1}^{3} \Delta y_{t}}{\sum_{t=1}^{T} y_{t-1}^{6}}
$$

Then the corresponding conventional $t$ statistic is given by

$$
t_{\hat{\delta}}=\frac{\hat{\delta}}{\sqrt{\operatorname{Var}(\hat{\delta})}}=\frac{\sum_{t=1}^{T} y_{t-1}^{3} \Delta y_{t}}{\sqrt{\hat{\sigma}^{2} \sum_{t=1}^{T} y_{t-1}^{6}}},
$$


where

$$
\hat{\sigma}^{2}=\frac{1}{T} \sum_{t=1}^{T}\left(\Delta y_{t}-\hat{\delta} y_{t-1}^{3}\right)^{2}
$$

is the usual estimator of the error variance.

To derive the asymptotics of $\hat{\delta}$ and $t_{\hat{\delta}}$, we have to be precise about the structure we are going to allow for the error term $\left\{v_{t}\right\}$. Kapetanios et al. (2003) only consider the $t$ statistic and show that for an i.i.d. sequence with finite second moments the limit distribution is given by

$$
t_{\hat{\delta}} \Rightarrow \frac{\frac{1}{4} W(1)^{4}-\frac{3}{2} \int_{0}^{1} W(r)^{2} d r}{\sqrt{\int_{0}^{1} W(r)^{6} d r}} .
$$

They also demonstrate that this asymptotic distribution is the same in the case of an autoregressive process of order $p$, when the auxiliary regression (5) is extended with lagged first differences, as in Said and Dickey (1984), to

$$
\Delta y_{t}=\delta y_{t-1}^{3}+\sum_{i=1}^{p} \rho_{j} \Delta y_{t-i}+\epsilon_{t}
$$

where $\epsilon_{t}$ is i.i.d. $\left(0, \sigma^{2}\right)$ under the null. Given that $p$ increases with the sample size in a suitable way, it can be shown that this also holds for general stationary and invertible ARMA processes.

In this paper, we will use a much more general framework based on the concept of strong mixing process as used in Hansen (1992). The main advantages of this approach is that besides its flexibility in terms of dependence structures, it also allows the possibility of unconditional or conditional heteroskedasticity, which is important for many economic applications. Furthermore, it also seems unintuitive to restrict the attention to linear error sequences in the general context of nonlinear time series.

More precisely, we make the following assumption about $\left\{v_{t}\right\}$ :

Assumption 2.1. For some $p>\beta>2,\left\{v_{t}\right\}$ is a zero mean, strong mixing sequence with mixing coefficients $\alpha_{m}$ of size $-p \beta /(p-\beta)$ and $\sup _{i \geq 1}\left\|v_{i}\right\|_{p}=$ $C<\infty$. In addition, $(1 / T) E\left(\left(\sum_{1}^{T} v_{i}\right)^{2}\right) \rightarrow \lambda^{2}>0$ as $T \rightarrow \infty$.

The basic idea behind that assumption is that there should be a trade-off between the existence of moments and the mixing decay rate in order to prevent single observations from being too influential. For example, if extreme realizations occur relatively often (as will be the case when relatively few moments are finite), then the effect of such observations should wear off at a faster rate (requiring a larger size). Assumption 2.1 thus assures that the functional central limit theorem (FCLT) and results regarding the convergence to stochastic intergrals will apply to certain normalized partial sums of $\left\{v_{t}\right\}$.

The parameter $\lambda^{2}$ is called the long-run variance. Note that while the second moments of $\left\{v_{t}\right\}$ are not assumed to be constant over time, a strong law of large numbers (McLeish 1975) asserts that

$$
\frac{1}{n} \sum_{i=1}^{n} v_{i}^{2} \stackrel{a . s}{\longrightarrow} \sigma^{2}=\lim _{n \rightarrow \infty} \frac{1}{n} \sum_{i=1}^{n} E\left(v_{i}^{2}\right) .
$$


Hence there is a parameter $\sigma^{2}$ that can be interpreted as the average error variance. When $\left\{v_{t}\right\}$ is a sequence of i.i.d. variables, we obviously have $\lambda^{2}=\sigma^{2}$.

Having defined the theoretical framework, we can now establish some convergence results that will be essential for the further asymptotic development.

Lemma 2.1. Let Assumption 2.1 hold with $\beta=6$ and $H_{0}$ be true. Then, as $T \rightarrow \infty$, we have:

$$
\begin{aligned}
& \text { (a) } T^{-2} \sum_{t=1}^{T} y_{t-1}^{2} \Rightarrow \lambda^{2} \int_{0}^{1} W(r)^{2} d r \\
& \text { (b) } T^{-4} \sum_{t=1}^{T} y_{t-1}^{6} \Rightarrow \lambda^{6} \int_{0}^{1} W(r)^{6} d r \\
& \text { (c) } T^{-2} \sum_{t=1}^{T} y_{t-1}^{3} v_{t} \Rightarrow \frac{1}{4} \lambda^{4} W(1)^{4}-\frac{3}{2} \sigma^{2} \lambda^{2} \int_{0}^{1} W(r)^{2} d r
\end{aligned}
$$

Proof. Both (a) and (b) are standard results that follow from the FCLT and the continuous mapping theorem (CMT), whereas (c) is a result of Theorem A.1 in the appendix, its subsequent remark, and the Itô formula.

Now we can start to discuss the properties of the quantities in (7)-(9). Our first concern is whether the estimates are consistent. This is ensured by the following theorem.

Theorem 2.1. Under the conditions of Lemma 2.1, we have

$$
\begin{aligned}
& \text { (a) } \hat{\delta} \stackrel{p}{\rightarrow} 0, \hat{\delta}=O_{p}\left(T^{2}\right) \\
& \text { (b) } \hat{\sigma}^{2} \stackrel{p}{\rightarrow} \sigma^{2}, \hat{\sigma}^{2}-\sigma^{2}=O_{p}(T)
\end{aligned}
$$

Proof. Part (a) can be shown by a comparison of the convergence rates of the nominator and denominator in $(7)$, which are $O_{p}\left(T^{-2}\right)$ and $O_{p}\left(T^{-4}\right)$, respectively. To prove (b), write

$$
\hat{\sigma}^{2}=\frac{1}{T} \sum_{t=1}^{T} v_{t}^{2}-\frac{2 \hat{\delta}}{T} \sum_{t=1}^{T} y_{t-1}^{3} v_{t}+\frac{\hat{\delta}^{2}}{T} \sum_{t=1}^{T} y_{t-1}^{6}
$$

and note that the first term converges to $\sigma^{2}$ in probability by (12), and the last two terms are both $O_{p}(T)$. Hence, the result follows.

Part (a) of the theorem implies that $\hat{\delta}$ converges to its true value zero under $H_{0}$ at rate $T^{2}$, which is even faster than the usual rate $T$ encountered in ordinary time series regressions with a unit root. Using this and the previous results, we can now derive the asymptotic distributions of the appropriately scaled version of $\hat{\delta}$ and of $t_{\hat{\delta}}$.

Theorem 2.2. Under the conditions of Lemma 2.1, we have

$$
\begin{aligned}
& \text { (a) } T^{2} \hat{\delta} \Rightarrow \frac{\frac{1}{4} \lambda^{4} W(1)^{4}-\frac{3}{2} \sigma^{2} \lambda^{2} \int_{0}^{1} W(r)^{2} d r}{\lambda^{6} \int_{0}^{1} W(r)^{6} d r} \\
& \text { (b) } t_{\hat{\delta}} \Rightarrow \frac{\frac{1}{4} \lambda^{4} W(1)^{4}-\frac{3}{2} \sigma^{2} \lambda^{2} \int_{0}^{1} W(r)^{2} d r}{\sqrt{\sigma^{2} \lambda^{6} \int_{0}^{1} W(r)^{6} d r}}
\end{aligned}
$$


Proof. Part (a) follows directly from Lemma 2.1 (b) and (c) and the CMT, whereas part (b) additionally requires Theorem 2.1 (b).

\section{The $Z_{N L}$ test}

Unfortunately, the limiting distributions derived in Theorem 2.2 depend on unknown nuisance parameters, and hence the respective test statistics cannot directly be used. We solve the problem by the semi-parametric approach proposed in Phillips (1987) and Phillips and Perron (1988). Without specifying an explicit model for $\left\{v_{t}\right\}$, we use consistent estimates of $\lambda^{2}$ and $\sigma^{2}$ to modify the test statistics in such a way that the parameters cancel each other asymptotically.

Let us assume for the moment that we have two such estimators, named $\hat{\lambda}^{2}$ and $\hat{\sigma}^{2}$, without worrying about their exact functional form. These consistent estimates can now be used to develop Phillips-Perron-type test statistics, whose limiting distributions do not depend on nuisance parameters.

Define

$$
Z_{N L}(\delta)=\hat{\lambda}^{2} T^{2}\left(\hat{\delta}-\frac{3}{2} \sum_{t=1}^{T} y_{t-1}^{2}\left(\hat{\lambda}^{2}-\hat{\sigma}^{2}\right)\left(\sum_{t=1}^{T} y_{t-1}^{6}\right)^{-1}\right)
$$

and

$$
Z_{N L}(t)=\frac{\hat{\sigma}}{\hat{\lambda}} t_{\hat{\delta}}-\frac{3}{2} \sum_{t=1}^{T} y_{t-1}^{2}\left(\hat{\lambda}^{2}-\hat{\sigma}^{2}\right)\left(\hat{\lambda}^{2} \sum_{t=1}^{T} y_{t-1}^{6}\right)^{-1 / 2},
$$

where the first term is a transformation of the OLS estimate $\hat{\delta}$ and the second term is one of the corresponding $t$-statistic $t_{\hat{\delta}}$. The respective asymptotic distributions of the two quantities are given by the following theorem:

Theorem 3.1. Under the conditions of Lemma 2.1, we have

$$
\begin{aligned}
& \text { a) } Z_{N L}(\delta) \Rightarrow \frac{\frac{1}{4} W(1)^{4}-\frac{3}{2} \int_{0}^{1} W(r)^{2} d r}{\int_{0}^{1} W(r)^{6} d r} \\
& \text { b) } Z_{N L}(t) \Rightarrow \frac{\frac{1}{4} W(1)^{4}-\frac{3}{2} \int_{0}^{1} W(r)^{2} d r}{\sqrt{\int_{0}^{1} W(r)^{6} d r}}
\end{aligned}
$$

Proof. Both (a) and (b) are a direct consequence of Theorem 2.2, Lemma 2.1 and the CMT.

As they are free of unknown parameters, these limiting distributions can now directly be used to obtain critical values for a test of $H_{0}$. A nice parallel to the standard linear Phillips-Perron tests is that the distributions coincide with the ones of the unmodified statistics $T^{2} \hat{\delta}$ and $t_{\hat{\delta}}$ when $\left\{v_{t}\right\}$ is an i.i.d. sequence and we thus have $\lambda^{2}=\sigma^{2}$ (see Theorem 2.2).

Figure 3.2 depicts the limit distributions of the two statistics under $H_{0}$. It turns out that the one of $Z_{N L}(\delta)$ is heavily left skewed, whereas the one of $Z_{N L}(t)$ is much more concentrated and only relatively mildly skewed. 


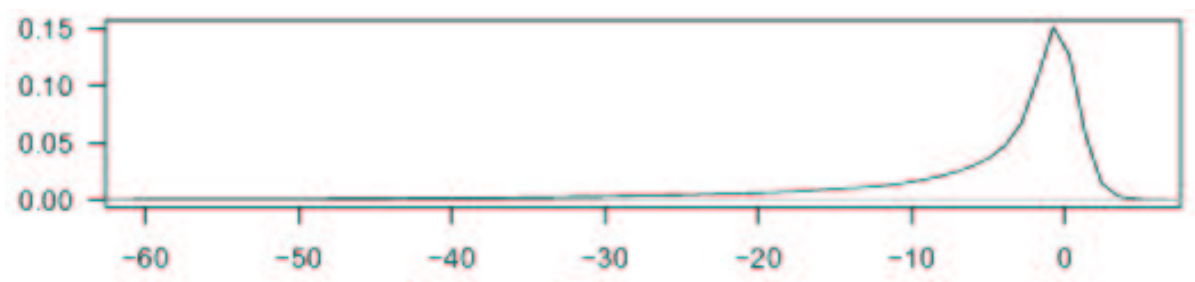

(a)

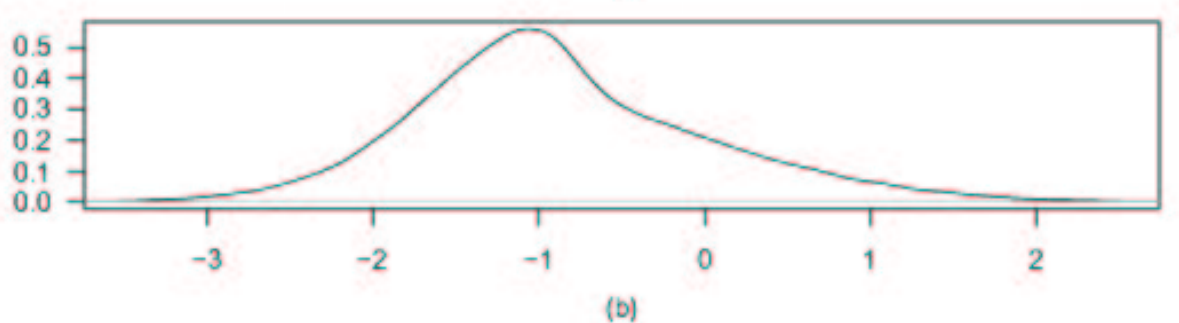

Figure 3.2: (a) Estimated probability density function of the $Z_{N L}(\delta)$ statistic. (b) Estimated probability density function of the $Z_{N L}(t)$ statistic.

Table 1: Asymptotic critical values of the $Z_{N L}(\delta)$ and $Z_{N L}(t)$ statistics

\begin{tabular}{l|rrr|rrr}
\hline \hline & \multicolumn{3}{|c|}{$Z_{N L}(\delta)$} & \multicolumn{3}{c}{$Z_{N L}(t)$} \\
Fractile (\%) & Case 1 & Case 2 & Case 3 & Case 1 & Case 2 & Case 3 \\
\hline 1 & -131.184 & -260.396 & -504.780 & -2.802 & -3.481 & -3.944 \\
5 & -50.834 & -121.848 & -279.798 & -2.202 & -2.934 & -3.404 \\
10 & -28.927 & -73.395 & -200.390 & -1.916 & -2.652 & -3.126 \\
\hline \hline
\end{tabular}

$\overline{\text { Cases refer to raw, demeaned, and demeaned and detrended data, respectively. }}$

Up to now, we have confined our analysis to stochastic processes with mean zero, which certainly limits the usefulness of the results for empirical applications. To accommodate for nonzero means and/or deterministic linear trends, we use the same minor modifications as in Kapetanios et al. (2003). In case of a nonzero mean, i.e. when our model is $x_{t}=\mu+y_{t}$, we use the demeaned data, $\hat{y}_{t}=x_{t}-\bar{x}$, where $\bar{x}$ is the sample mean. The new limiting distributions are basically the same as in Theorem 3.1, but with $W(r)$ replaced by $W^{*}(r)$, a standard de-meaned Wiener process on $[0,1]$. Similarly, when we assume both a nonzero mean and a linear trend, i.e. when our model is $x_{t}=\mu+\beta t+y_{t}$, we will use the demeaned and detrended data, $\hat{y}_{t}=x_{t}-\hat{\mu}-\hat{\beta}$, where $\hat{\mu}$ and $\hat{\beta}$ are the simple OLS estimates. In this case, $W(r)$ is replaced by $W^{\#}(r)$, a demeaned and detrended standard Wiener process defined on $[0,1]$.

Asymptotic critical values for these three constellations, termed case 1, case 2 and case 3 , are given in Table 1 . They were obtained by simulating the respective statistics with $T=1,000$ observations and 50,000 replications.

Finally, we have to discuss the estimation of the nuisance parameters. In the case of $\sigma^{2}$, the usual estimator $\hat{\sigma}^{2}$ has already shown to be consistent under the null hypothesis in Theorem 2.1 (b). There is also a large number of long-run variance estimators proposed in the literature (see Den Haan and Levin (1997) for a comprehensive review). While roughly speaking any consistent estimator 
will do the job, in this paper we will use the Newey-West estimator (Newey and West 1987)

$$
\hat{\lambda}^{2}=\hat{\Gamma}(0)+2 \sum_{i=1}^{T-1} w\left(\frac{i}{l_{T}}\right) \hat{\Gamma}(i),
$$

where $\hat{\Gamma}(\tau)=T^{-1} \sum_{t=\tau+1}^{T} \hat{u}_{t} \hat{u}_{t-\tau}, w(\cdot)$ is the Bartlett kernel and the lag truncation parameter $l_{T}$ is set at $\left\lfloor 4(T / 100)^{2 / 9}\right\rfloor$. While the specific functional for the estimator is not important for the asymptotic development, it may well affect the tests' finite sample performance. It should therefore be stressed, that our particular choice is rather an ad hoc decision than based on theoretical considerations. The question whether other approaches might be more favourable in our context is beyond the scope of this paper.

\section{Simulation results}

In order to assess the properties of the new test statistics and to compare their performance with the procedures proposed by Kapetanios et al. (2003), Phillips and Perron (1988) and Said and Dickey (1984), we conduct a series of simulation experiments. For brevity, we only consider the first of the three cases described above, with the data being neither demeaned nor detrended. Although the power is lower for the last two cases, the performances of the tests relative to each other are similiar.

Our first concern is the size of the tests in finite samples, and thus we construct a null model with moving average errors by

$$
y_{t}=y_{t-1}+v_{t} \quad \text { with } \quad v_{t}=e_{t}+\rho e_{t-1}
$$

where the $e_{t}$ are i.i.d $N(0,1)$, and we consider $\rho=\{0, \pm 0.2, \pm 0.5, \pm 0.8\}$.

To evaluate the power of the two testing procedures against a globally meanreverting $\operatorname{ESTAR}(1)$, we generate the data by

$$
\Delta y_{t}=\gamma y_{t-1}\left(1-\exp \left(-\theta y_{t-1}^{2}\right)\right)+v_{t} \quad \text { with } \quad v_{t}=e_{t}+\rho e_{t-1},
$$

and consider $\theta=\{0.01,0.05,0.1,1\}$ and $\gamma=\{-1.5,-1,-0.5,-0.1\}$. We also used various specifications of the error process, but for brevity only the cases where $\rho=\{0,0.5\}$ and the $e_{t}$ are i.i.d $N(0,1)$ are reported here.

For both size and power simulations, the nominal level of the tests is $5 \%$, the sample size being considered is $T=\{50,100\}$, and the number of replications is set at 10,000 .

In contrast to Kapetanios et al. (2003), we do not assume that the DGP is known when applying the tests to the data. In particular, we determine the number of augmentations used for the $t_{N L}$ and ADF tests by minimizing the Schwartz Information Criterion SIC in the respective regressions. While the question whether this approach is efficient or not is beyond the scope of this paper, it certainly gives a more realistic impression of the properties of the tests than just using the "true" order.

Table 2 shows the results on the size of the various tests. For $\rho=\{0,0.2,0.5\}$ all sizes are generally close to the nominal level of $5 \%$. We also observe the tendency to overreject the null somewhat when $\rho=\{-0.2,0.8\}$. For large 
Table 2: The size of alternative test

\begin{tabular}{lcccccc}
\hline \hline Error Process & $Z_{N L}(\delta)$ & $Z_{N L}(t)$ & $t_{N L}$ & $Z(\alpha)$ & $Z(t)$ & ADF \\
\hline $\mathrm{T}=50$ & & & & & & \\
$\rho=0.8$ & 0.090 & 0.091 & 0.077 & 0.085 & 0.080 & 0.072 \\
$\rho=0.5$ & 0.066 & 0.068 & 0.077 & 0.067 & 0.062 & 0.069 \\
$\rho=0.2$ & 0.040 & 0.044 & 0.044 & 0.047 & 0.046 & 0.050 \\
$\rho=0$ & 0.036 & 0.056 & 0.054 & 0.049 & 0.048 & 0.050 \\
$\rho=-0.2$ & 0.053 & 0.094 & 0.111 & 0.077 & 0.083 & 0.106 \\
$\rho=-0.5$ & 0.160 & 0.290 & 0.219 & 0.256 & 0.283 & 0.230 \\
$\rho=-0.8$ & 0.507 & 0.713 & 0.379 & 0.651 & 0.706 & 0.466 \\
$\mathrm{~T}=100$ & & & & & & \\
$\rho=0.8$ & 0.093 & 0.091 & 0.056 & 0.089 & 0.083 & 0.051 \\
$\rho=0.5$ & 0.066 & 0.065 & 0.073 & 0.066 & 0.060 & 0.064 \\
$\rho=0.2$ & 0.046 & 0.047 & 0.046 & 0.048 & 0.047 & 0.046 \\
$\rho=0$ & 0.044 & 0.056 & 0.051 & 0.052 & 0.051 & 0.050 \\
$\rho=-0.2$ & 0.069 & 0.094 & 0.112 & 0.084 & 0.087 & 0.104 \\
$\rho=-0.5$ & 0.203 & 0.284 & 0.134 & 0.237 & 0.252 & 0.152 \\
$\rho=-0.8$ & 0.719 & 0.811 & 0.289 & 0.683 & 0.722 & 0.385 \\
\hline \hline
\end{tabular}

Note: Data under the null is generated by (17).

negative values of $\rho$, however, size distortions are extreme for all of the six tests, with the ADF type test being a little less affected. This is a well known result for the linear unit root tests, which was already mentioned by Phillips and Perron (1988) in their original work. The problem seems to persist in the nonlinear case, so that none of the tests is recommendable when the error process almost has a negative moving average unit root.

Results of the power simulations are presented in Table 3 and 4 . The finding of Kapetanios et al. (2003) was that their $t_{N L}$ test performed better than the $\mathrm{ADF}$ for small values of $\theta$. In that case, the process is highly persistent. When $\theta$ grows, however, the average values of the transition function $G\left(y_{t-1}, \theta\right)$ get closer to one and the process more and more resembles a stable, linear AR(1). So eventually, the power of the ADF dominates. ${ }^{3}$ In their empirical application, they find that estimates of $\theta$ are usually indeed quite small in the case of foreign exchange rates, and conclude that their test should be used in that situation. Our simulations confirm these findings and show that basically the same holds for the two linear Phillips-Perron tests $Z(\alpha)$ and $Z(t)$. Their performance turns out to be marginally worse than the ADF's one, but they beat the $t_{N L}$ provided that $\theta$ is sufficiently large.

All of the six tests have in common that their power is close to one when $\gamma$ is small. This comes as no surprise, as in these cases the process is less persistent.

Regarding our two new tests, we make the following observations. First, comparing $Z_{N L}(\delta)$ and $Z_{N L}(t)$, we note that the power of the latter dominates the one of the former, particularly when $\theta$ is small. In addition, $Z_{N L}(\delta)$ performs similar or worse than all its linear competitors, leading to the conclusion that this test should not be applied in practice. This comes as a surprise, since unit root tests based directly on regression coeficients are usually found to be more

\footnotetext{
${ }^{3}$ Note that $\theta$ is not a scale free parameter and that thus it is difficult to say which values are actually "small" or "large".
} 


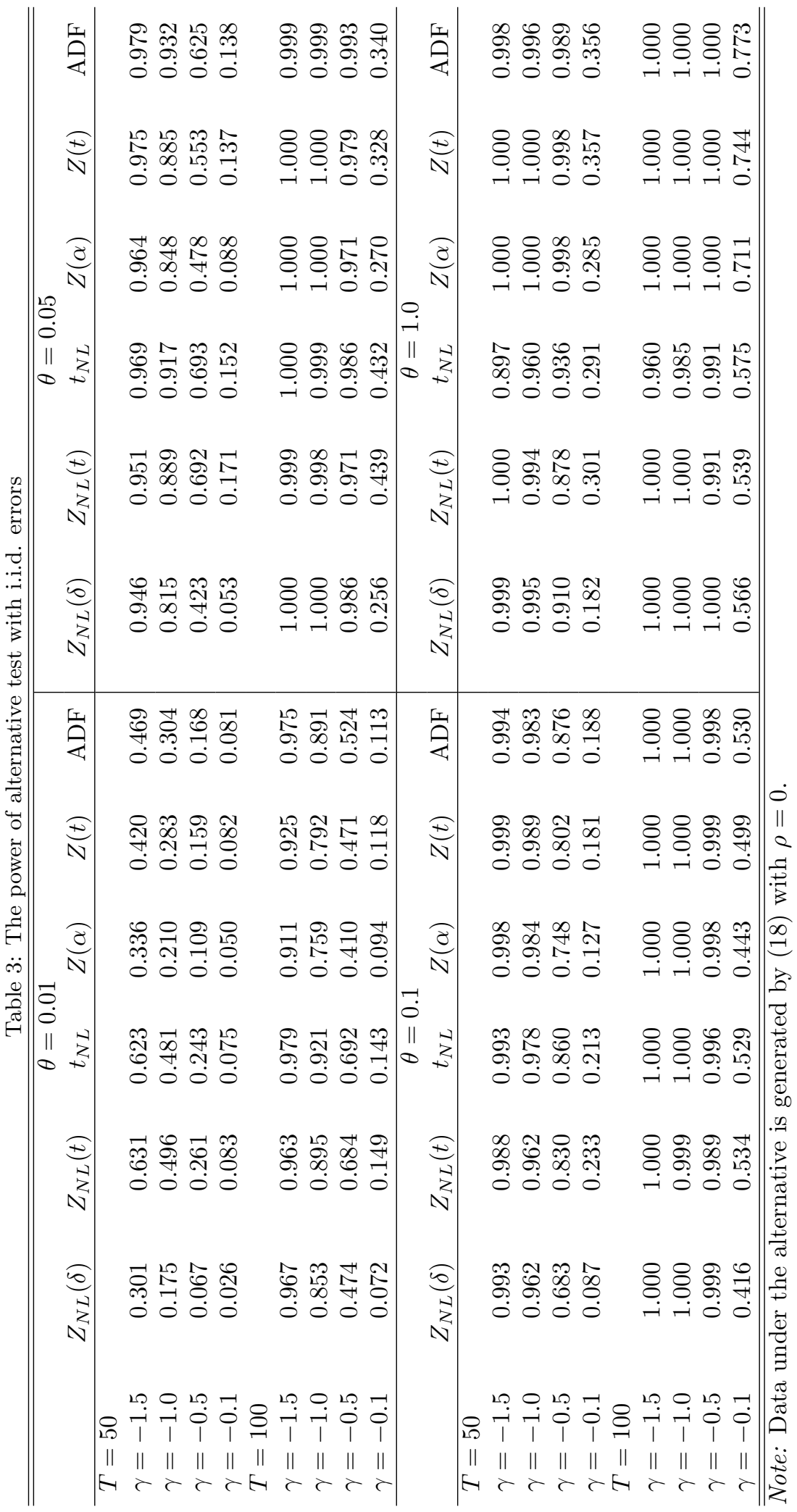




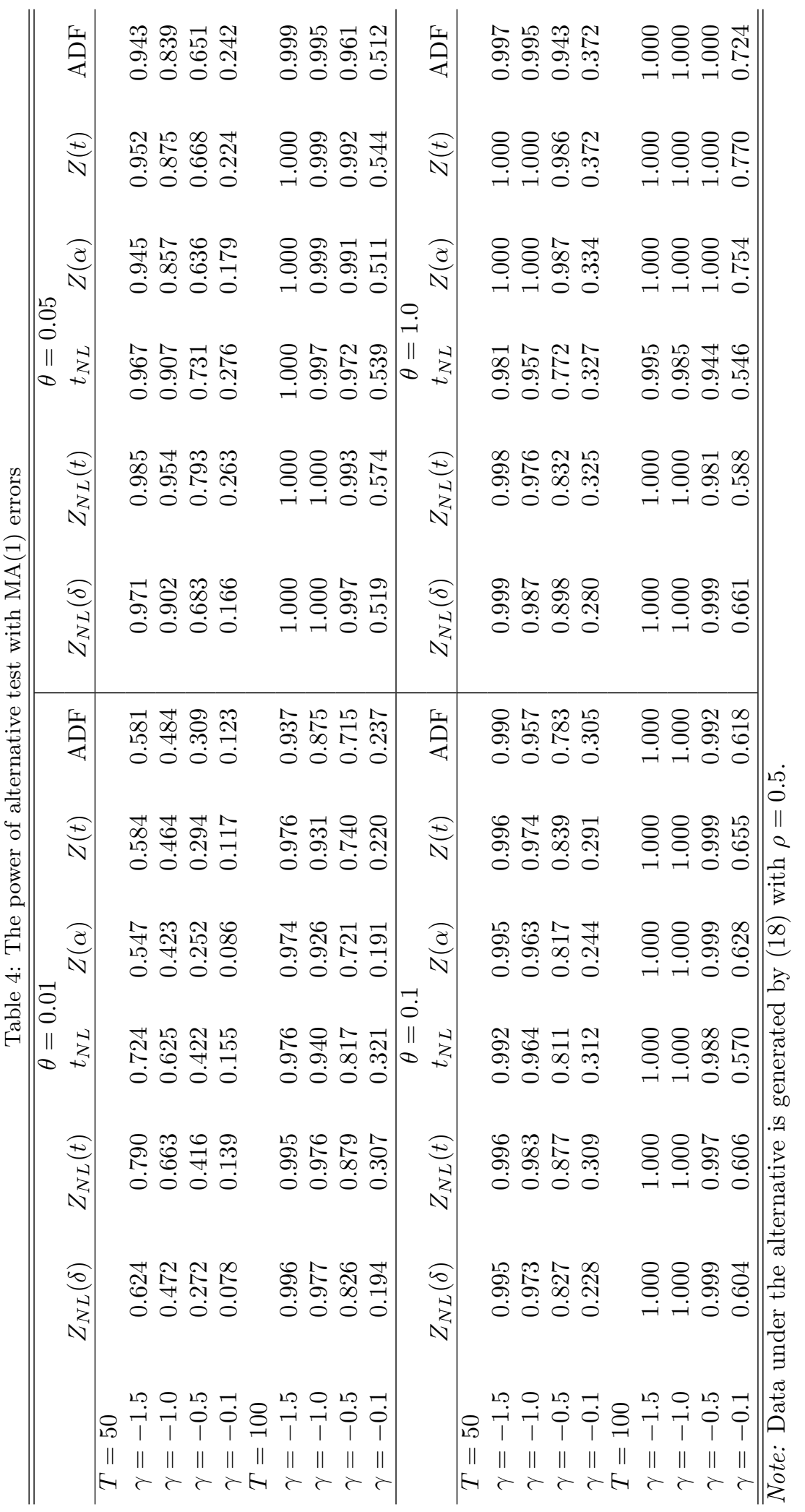




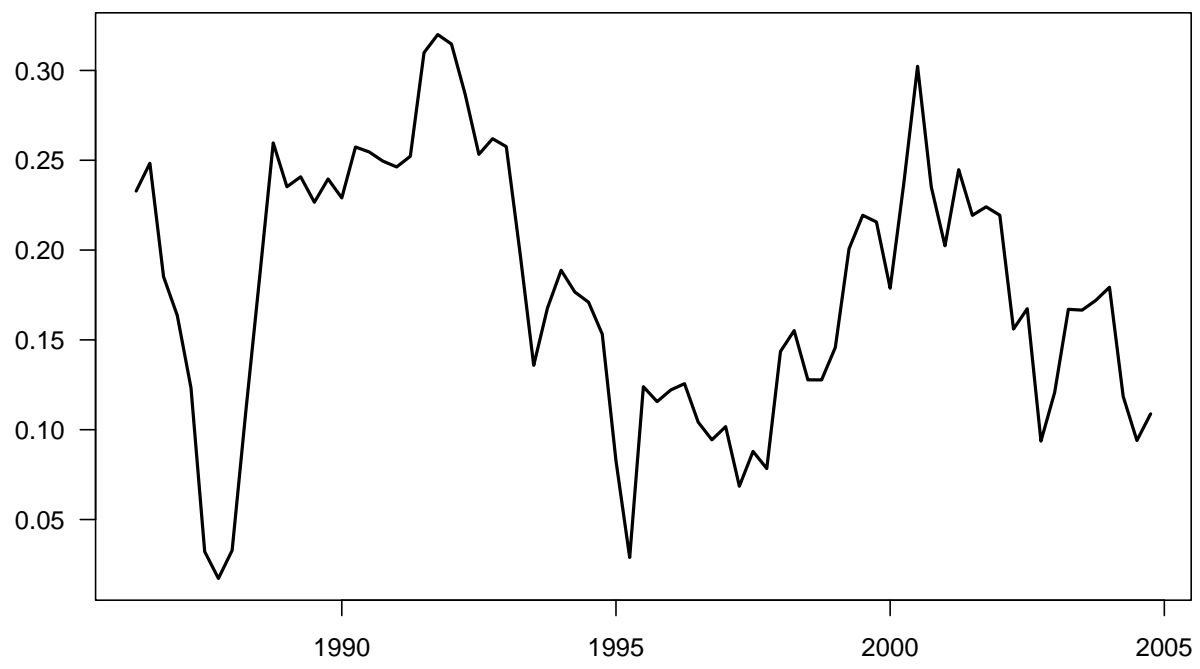

Figure 5.3: Quarterly (log) real bilateral exchange rate of the Australian and New Zealand Dollar.

powerful than the ones based on $t$-ratios (Phillips and Xiao 1998).

Secondly, it emerges from Table 4 that $Z_{N L}(t)$ is marginally more powerful than $t_{N L}$ when the error sequence is an MA(1). For i.i.d. errors, Table 3 shows that both test perform equally well in terms of power for all parameter constellations considered here. The small differences between the respective values are probably the result of simulation errors.

Note that in the latter case both the augmentations and the semiparametric correction of the test statistics would not be necessary under normal circumstances. But since we consider only an approximation, the regression residuals will exhibit serial correlation due to the inclusion of the remainder from the Taylor series approximation. Both the $Z_{N L}(t)$ and the $t_{N L}$, however, seem to handle that problem equally well.

\section{Empirical Application: PPP}

As an empirical application of the unit root test developed in the previous chapter, consider the case of real exchange rates, defined as

$$
Q_{t}=S_{t} \cdot \frac{P_{t}^{*}}{P_{t}}
$$

where $S_{t}$ is the nominal exchange rate and $P_{t}^{*}, P_{t}$ are the foreign and domestic price levels at time $t$, respectively. It is common, however, to use the logarithmic form

$$
q_{t}=s_{t}+p_{t}^{*}-p_{t},
$$

where all lower case variables are the log of the respective upper case counterpart. As outlined in the introduction, the theory of purchasing power parity, or $\mathrm{PPP}$, predicts that the time series $q_{t}$ should be mean reverting, since short run 
Table 5: Test statistics and corresponding $p$-values.

\begin{tabular}{lrrrr}
\hline \hline & ADF & $Z(t)$ & $t_{N L}$ & $Z_{N L}(t)$ \\
\hline Statistic & -2.3357 & -2.5842 & -3.3622 & -3.1861 \\
$p$-value & 0.1600 & 0.0952 & 0.0145 & 0.0247 \\
\hline \hline
\end{tabular}

deviations from parity should be arbitraged away by market forces in the long run. Note that this is equivalent to saying that $s_{t}, p_{t}^{*}$ and $p_{t}$ are cointegrated with known cointegration vector $(1,1,-1)^{\prime}$. Standard unit root test however are often not able to reject the null of a unit root in $q_{t}$, which has been attributed to possible nonlinearities in the process. We expect our $Z_{N L}(t)$ test (and the similarly constructed $t_{N L}$ ) to be more powerful than the usual procedures in this case.

For our illustration, we consider the case of Australia and New Zealand. Due to their geographical situation and common history as British colonies, the countries have strong economic ties. Furthermore, with the 1983 Closer Economic Relations Trade Agreement (known as CER), most trade restrictions have successively been removed. All this makes it more likely to find evidence in support of PPP.

Our dataset, obtained from the IMF's International Financial Statistics Online service, contains the nominal exchange rate of the Australian to the New Zealand Dollar, together with the consumer price indices (CPI) of the respective countries as proxies of the price level. Observations were sampled at quarterly intervals, starting in 1986, after which both currencies were floating freely, and running to 2004. The resulting real exchange rate is displayed in Figure 5.3. It can be observed that the two countries' series has roughly stayed constant over the inspected time period, although there have been substantial fluctuations.

We applied four unit root tests to each of the time series: the linear ADF and PP tests based on the $t$-statistic, the $t_{N L}$ from Kapetanios et al. (2003), and our $Z_{N L}(t)$ test. Other procedures discussed earlier were omitted since the simulation results in the previous section suggest that their finite sample properties are at most similar, if not inferior, compared to the others. Since there is no theoretical justification for a linear trend in real exchange rates, we included only an intercept in the regression for the two linear tests, and used demeaned data for the two nonlinear ones. The resulting test statistics and corresponding simulated $p$-values are presented in Table 5 .

As one can see, the evidence in favour of PPP from the linear tests is not really compelling. In contrast, the two nonlinear ones strongly reject the hypothesis of a unit root in the real exchange rate, in line with economic theory.

In addition to that, we estimated the ESTAR model from (3) for $y_{t}-\mu$ by nonlinear least squares (NLS), yielding

$$
\Delta y_{t}=\underset{(0.1019)}{0.2473}\left(y_{t-1}-\underset{(0.0173)}{0.1880)}\left(1-\exp \left(-\underset{(295.8460)}{207.6174}\left(y_{t-1}-\underset{(0.0173)}{0.1880}\right)^{2}\right)\right)+\hat{v}_{t},(21)\right.
$$

where the numbers in parentheses are the corresponding standard errors. A further analysis of the residuals shows no sign of serial dependence, which demonstrates that the model is well specified. It is also strongly favoured over a linear AR model with intercept by both the AIC and SIC.

As pointed out by van Dijk et al. (2002), the large standard error of the estimate of $\theta$ should not be interpreted as evidence against nonlinearity, since 
the asymptotic distribution is nonstandard when $\theta=0$ due to the identification problem. In fact, the reason is that the residual sum of squares function is rather flat with respect to $\theta$, and thus a precise estimate is hard to obtain.

\section{Concluding Remarks}

In various fields of economics, theory suggests processes to be stationary or at least mean-reverting. A prime example is the theory of purchasing power parity, which we use to motivate our study. The frequent inability of standard testing procedures to reject the null of a unit root in these cases has long caused discomfort amongst applied researchers. Recently, this has been attributed to a lack of power of those tests against nonlinear alternatives.

This paper contributes to the literature in this field by proposing two new test statistics in the framework of the exponential smooth transition autoregressive (ESTAR) model. We derive their non-standard limit distributions analytically and assess the finite sample properties through a simulation study. As a result, one of the tests turns out to have slightly favourable properties over a procedure suggested by Kapetanios et al. (2003), the only one available in this context so far. In a small empirical application to real exchange rates we demonstrate that the approach is at least a promising one to solve the puzzle of purchasing power parity.

As always, there is some room for improvements. First, the model considered under the alternative is still somewhat restrictive. One might want to allow for a more complex structure, involving a higher order of lagged variables, or a non-constant mean or time trend, changing smoothly with the regimes. Other transition functions, such as the logistic one, would be interesting to consider as well, as they allow for asymmetric adjustment.

Second, solving the identification problem under the null hypothesis by other means than linearization could be considered. The issue is discussed, inter alia, by Andrews and Ploberger (1994) and Hansen (1996). In a much more general context than the one presented in this paper, they suggest calculating the test statistics as a function of the unidentified nuisance parameters, and then use the supremum or average over all possible values to conduct inference. The respective limit distributions will typically be non-standard and critical values are again obtained via simulation. Theoretical considerations suggest that these procedures will yield a better test. However, our approach presented in this paper has the advantage of greater conceptual and computational simplicity.

\section{A Mathematical Appendix}

For the following convergence theorem, consider a time series of the form

$$
y_{i}=y_{i-1}+v_{i}, \quad i=1 \ldots n,
$$

and define $V_{t}=\sum_{i=1}^{t} v_{i}$ and $V_{n}(s)=n^{-1 / 2} V_{\lfloor n s\rfloor}$ for $s \in[0,1]$.

Theorem A.1. Let $\Lambda=\lim _{n \rightarrow \infty} n^{-1} \sum_{i=1}^{n} \sum_{j=i+1}^{\infty} E\left(v_{i} v_{j}\right)$. If Assumption 2.1 holds with $\beta=6$, then

$$
\int_{0}^{s} V_{n}(r)^{3} d V_{n}(r) \Rightarrow \int_{0}^{s} B(r)^{3} d B(r)+3 \Lambda \int_{0}^{s} B^{2}(r) d r
$$


where $B=\lambda W$ is a Brownian motion with variance $\lambda^{2}$.

Proof. To begin with, recall that by the FCLT $V_{n}(\cdot) \Rightarrow B(\cdot)$ and hence $V_{n}^{3}(\cdot) \Rightarrow$ $B^{3}(\cdot)$ by the CMT. The following derivation extensively uses Theorem 3.1 to 3.3 from Hansen (1992), which will be refered to as $\mathrm{H} 1$ to H3, respectively.

Let $\mathcal{F}_{t}=\sigma\left(v_{i}: i \leq t\right)$ be the smallest sigma-field containing the past history of $\left\{v_{t}\right\}$, and define

$$
\begin{aligned}
\epsilon_{i} & =\sum_{k=0}^{\infty}\left(E\left(v_{i+k} \mid \mathcal{F}_{i}\right)-E\left(v_{i+k} \mid \mathcal{F}_{i-1}\right)\right), \\
z_{i} & =\sum_{k=1}^{\infty} E\left(v_{i+k} \mid \mathcal{F}_{i}\right) .
\end{aligned}
$$

Then $\epsilon_{i}+z_{i-1}-z_{i}=E\left(v_{i} \mid \mathcal{F}_{i}\right)=v_{i}$ and it is easy to show that $\left\{\epsilon_{t}, \mathcal{F}_{t}\right\}$ is a martingale difference sequence. With $Y_{n}(s)=n^{-1 / 2} \sum_{i=1}^{\lfloor n s\rfloor} \epsilon_{i}$, one directly obtains the equality

$$
\int_{0}^{s} V_{n}^{3}(r) d V_{n}(r)=\int_{0}^{s} V_{n}^{3}(r) d Y_{n}(r)+\Lambda_{n}^{*}(s),
$$

where

$$
\Lambda_{n}^{*}(s)=\frac{1}{n^{2}} \sum_{i=1}^{\lfloor n s\rfloor}\left(V_{i}^{3}-V_{i-1}^{3}\right) z_{i}-\frac{1}{n^{2}} V_{\lfloor n s\rfloor}^{3} z_{\lfloor n s\rfloor+1} .
$$

Then, by H1, we obtain

$$
\int_{0}^{s} V_{n}^{3}(r) d Y_{n}(r) \Rightarrow \int_{0}^{s} B^{3}(r) d B(r)
$$

Now consider the bias term

$$
\begin{aligned}
\Lambda_{n}^{*}(s) & =\frac{1}{n^{2}} \sum_{i=1}^{\lfloor n s\rfloor}\left(V_{i}^{3}-V_{i-1}^{3}\right) z_{i}-\frac{1}{n^{2}} V_{\lfloor n s\rfloor}^{3} z_{\lfloor n s\rfloor+1} \\
& =\frac{1}{n^{2}} \sum_{i=1}^{\lfloor n s\rfloor}\left(3 V_{i-1}^{2} v_{i}+3 V_{i-1} v_{i}^{2}+v_{i}^{3}\right) z_{i}+o_{p}(1) \\
& =\frac{3}{n^{2}} \sum_{i=1}^{\lfloor n s\rfloor} V_{i-1}^{2} v_{i} z_{i}+\frac{3}{n^{2}} \sum_{i=1}^{\lfloor n s\rfloor} V_{i-1} v_{i}^{2} z_{i}+\frac{1}{n^{2}} \sum_{i=1}^{\lfloor n s\rfloor} v_{i}^{3} z_{i}+o_{p}(1)
\end{aligned}
$$

First, by the triangle inequality and Hölder's inequality,

$$
\begin{aligned}
E \sup _{0 \leq s \leq 1}\left|\frac{1}{n^{2}} \sum_{i=1}^{\lfloor n s\rfloor} v_{i}^{3} z_{i}\right| & \leq E \sup _{0 \leq s \leq 1} \frac{1}{n^{2}} \sum_{i=1}^{\lfloor n s\rfloor}\left|v_{i}^{3} z_{i}\right| \\
& \leq \frac{1}{n^{2}} \sum_{i=1}^{n} E\left|v_{i}^{3} z_{i}\right| \\
& \leq \frac{1}{n^{2}} \sum_{i=1}^{n}\left\|v_{i}^{3}\right\|_{\frac{4}{3}}\left\|z_{i}\right\|_{4} \\
& \leq \frac{1}{n^{2}} \sum_{i=1}^{n}\left\|v_{i}\right\|_{4}^{3}\left\|z_{i}\right\|_{4} \rightarrow 0
\end{aligned}
$$


as $n \rightarrow \infty$, since by Assumption 2.1 and the proof of H1, respectively, both $\left\|v_{i}\right\|_{4}$ and $\left\|z_{i}\right\|_{4}$ are bounded.

Second, set $e_{i}=v_{i} z_{i}-\Lambda$, which by $\mathrm{H} 2$ satisfies the conditions of $\mathrm{H} 3$, yielding

$$
\sup _{0 \leq s \leq 1}\left|\frac{1}{n^{2}} \sum_{i=1}^{\lfloor n s\rfloor} V_{i-1}^{2} e_{i}\right| \stackrel{p}{\rightarrow} 0
$$

and hence we have

$$
\begin{aligned}
\frac{3}{n^{2}} \sum_{i=1}^{\lfloor n s\rfloor} V_{i-1}^{2} v_{i} z_{i} & =\frac{3}{n^{2}} \sum_{i=1}^{\lfloor n s\rfloor} V_{i-1}^{2} \Lambda+\frac{3}{n^{2}} \sum_{i=1}^{\lfloor n s\rfloor} V_{i-1}^{2} e_{i} \\
& \Rightarrow 3 \Lambda \int_{0}^{s} B^{2}(r) d r
\end{aligned}
$$

Finally,

$$
\begin{aligned}
E \sup _{0 \leq s \leq 1}\left|\frac{3}{n^{2}} \sum_{i=1}^{\lfloor n s\rfloor} V_{i-1} v_{i}^{2} z_{i}\right| & \leq E \sup _{0 \leq s \leq 1} \frac{3}{n^{2}} \sum_{i=1}^{\lfloor n s\rfloor}\left|V_{i-1} v_{i}^{2} z_{i}\right| \\
& \leq \frac{3}{n^{2}} \sum_{i=1}^{n} E\left|V_{i-1} v_{i}^{2} z_{i}\right| \\
& \leq \frac{3}{n^{2}} \sum_{i=1}^{n}\left\|V_{i-1}\right\|_{2}\left\|v_{i}^{2} z_{i}\right\|_{2} \rightarrow 0
\end{aligned}
$$

as $n \rightarrow \infty$. To see this, note that by Hölder's inequality

$$
\left\|v_{i}^{2} z_{i}\right\|_{2}=\left(E\left|v_{i}^{4} z_{i}^{2}\right|\right)^{1 / 2} \leq\left\|v_{i}\right\|_{6}^{2}\left\|z_{i}\right\|_{6}
$$

and thus is bounded. Furthermore,

$$
\begin{aligned}
\frac{3}{n^{2}} \sum_{i=1}^{n}\left\|V_{i-1}\right\|_{2} & =\frac{3}{n^{2}} \sum_{i=1}^{n}\left(E\left(V_{i-1}^{2}\right)\right)^{1 / 2} \\
& =\frac{3}{n^{3 / 2}} \sum_{i=1}^{n}\left(\frac{1}{n} E\left(V_{i-1}^{2}\right)\right)^{1 / 2} \\
& \leq \frac{3}{n^{3 / 2}} \sum_{i=1}^{n}\left(\frac{1}{n} E\left(V_{n}^{2}\right)\right)^{1 / 2} \rightarrow 0,
\end{aligned}
$$

as $n \rightarrow \infty$, since $n^{-1} E\left(V_{n}^{2}\right)$ is $O(1)$ and thus bounded.

REMARK: Note that since $E\left(V_{n}\right)=0$ for all $n$,

$$
\begin{aligned}
\lambda^{2} & =\lim _{n \rightarrow \infty} \operatorname{Var}\left(\frac{1}{\sqrt{n}} V_{n}\right) \\
& =\lim _{n \rightarrow \infty}\left(\frac{1}{n} \sum_{i=1}^{n} E\left(v_{i}^{2}\right)+\frac{2}{n} \sum_{i=1}^{n-1} \sum_{j=i+1}^{n} E\left(v_{i} v_{j}\right)\right) \\
& =\sigma^{2}+2 \Lambda
\end{aligned}
$$




\section{References}

Andrews, D. W. K. and Ploberger, W.: 1994, Optimal tests when a nuisance parameter is present only under the alternative, Econometrica 62(6), 13831414 .

Baum, C. F., Barkoulas, J. T. and Caglayan, M.: 2001, Nonlinear adjustment to purchasing power parity in the post-bretton woods era, Journal of International Money and Finance 20(3), 379-399.

Davies, R.: 1987, Hypothesis testing when a nuisance parameter is present only under the alternative, Biometrika 74(1), 33-43.

Den Haan, W. and Levin, A.: 1997, A practitioner's guide to robust covariance matrix estimation, in G. Maddala and C. Rao (eds), Handbook of Statistics 15, Elsevier, Amsterdam, pp. 299-342.

Dumas, B.: 1992, Dynamic equilibrium and the real exchange rate in a spatially separated world, Review of Financial Studies 5(2), 153-180.

Hansen, B. E.: 1992, Convergence to stochastic integrals for dependent heterogeneous processes, Econometric Theory 8, 489-500.

Hansen, B. E.: 1996, Inference when a nuisance parameter is not identified under the null hypothesis, Econometrica 64(2), 413-430.

Kapetanios, G., Shin, Y. and Snell, A.: 2003, Testing for a unit root in the nonlinear star framework, Journal of Econometrics 112(2), 359-379.

Luukkonen, R., Saikkonen, P. and Teräsvirta, T.: 1988, Testing linearity against smooth transition autoregressive models, Biometrica 75, 491-499.

McLeish, D. L.: 1975, A maximal inequality and dependent strong laws, Annals of Probability 3, 829-839.

Michael, P., Nobay, A. R. and Peel, D. A.: 1997, Transactions costs and nonlinear adjustment in real exchange rates: An empirical investigation, Journal of Political Economy 105(4), 862-79.

Newey, W. and West, K.: 1987, A simple, positive semi-definite, heteroskedasticity and autocorrelation consistent covariance matrix, Econometrica $\mathbf{5 5}(3), 703-708$.

Phillips, P. C. B.: 1987, Time series regression with a unit root, Econometrica $\mathbf{5 5}(2), 277-301$.

Phillips, P. C. B. and Perron, P.: 1988, Testing for a unit root in time series regression, Biometrika 75(2), 335-346.

Phillips, P. C. B. and Xiao, Z.: 1998, A primer on unit root testing, Journal of Economic Surveys 12(5), 423-69.

Said, S. and Dickey, D.: 1984, Testing for unit roots in autoregressive moving average of unknown order, Biometrika 71, 559-607. 
Sarno, L.: 2000, Real exchange rate behavior in the middle east: a reexamination, Economics Letters 66(2), 127-136.

Sercu, P., Uppal, R. and Van Hulle, C.: 1995, The exchange rate in the presence of transaction costs: Implications for tests of purchasing power parity, Journal of Finance 50(4), 1309-1319.

Taylor, M. P., Peel, D. and Sarno, L.: 2001, Nonlinear mean-reversion in real exchange rates: Towards a solution to the purchasing power parity puzzles, International Economic Review 42, 1015-1042.

van Dijk, D., Teräsvirta, T. and Franses, P. H.: 2002, Smooth transition autoregressive models - a survey of recent developments, Econometric Reviews 21(1), 1-47. 